\title{
Smart Modeling of Maritime Vessels
}

\author{
Przemyslaw Gilski and Jacek Stefanski \\ Faculty of Electronics, Telecommunications and Informatics, Gdansk University of Technology, Gdansk 80-233, Poland
}

\begin{abstract}
Currently, the market offers many visualization tools available to graphic designers, engineers, managers and academics working on maritime environments. The practice of visualization involves making and manipulating images that convey novel phenomena and ideas. Visual communication, together with virtual reality environments, is an emerging and rapidly evolving discipline. It brings great advantage over written word or voice alone, as visual sense is by far the most dominant component of human sensory perception. A new smart approach for modeling maritime vessels is described in this paper. Various ways of making modeling relevant for work within the field of maritime management, training and education are discussed.
\end{abstract}

Key words: Computer graphics, education, geographic information systems, marine technology, virtual reality.

\section{Introduction}

A significant progress has been made in the area of modeling and simulating. However, little attention has been given to smart and efficient ways of designing maritime vessels. More technical work on integrating plans and scenes is required for planning and management of vessels for various training or decision-making purposes.

Visualization is one of the main tools available for professionals involved in academic education, training and maritime planning context. A case point is the role that virtual reality environments play in planning and designing of maritime vessels.

Maritime vessel planning and simulation faces different communication challenges. Visualization techniques such as maps, graphical displays and virtual reality, are particularly important. In this case, environments such as this are unfamiliar to the general public.

Graphic designers and engineers often obtain information about the physical world and use it to improve foresight. It is also advisable to make it easier for students and academics to examine their own short-term and long-term features. This article

Corresponding author: Przemyslaw Gilski, M.Sc. E.Eng., $\mathrm{Ph} . \mathrm{D}$. student, research fields: computer graphics and wireless communication. describes methods of planning and modeling maritime vessels, from an engineer's perspective, in a smart and efficient way. It discusses communication tools for teachers and students. Ways of delivering the visual content referring to a full range of imagery, including photos and video, are also discussed.

\section{Visual Communication}

It is believed, that seeing is knowing. Therefore, research on how images are perceived and how visual phenomena influence the viewer is a crucial factor. The practice of modeling and visualization involves making and manipulating images in such a way, which convey novel phenomena and ideas, being a great advantage over written word or voice alone. The visual sense is by far the most dominant component of human sensory perception [1].

Most recent work on visualization techniques promotes expanding the sense of the visual side of all types of representation, including television, film and photographs across different fields, as the broadest range of representation possible. It ranges from maps to other representations of data like graphs or tables. Of course, environmental visualization can be multi-dimensional, including manipulated scenes in a virtual reality scenery [2].

Images play a major role in popular media. Scholars 
work on analyzing how different types of representations influence the viewer. Such analyses have focused on a broad range of media campaigns [3-5].

\subsection{GIS and Modeling}

Communication between the scientific and management communities can be a difficult process, further complicated by the nature of the goals of scientists and practitioners. Scientists are busy researching topics that are current and up to date, whereas practitioners are often working to implement the goals of their clients. In the latter case, goals are short-term, whereas for scientists, time is needed to conduct experiments and write about them.

Of course, advanced GIS (Geographic Information System) technologies are making the combinations of maps, plans or blueprints much easier. However, despite the ease of which one can migrate from one another, the simultaneous presentation is rather crude. Once the viewer is oriented, e.g. using satellite navigation, the location on a map will become ambiguous.

The main role of GIS systems is to effectively communicate with the user, by using optimal sizes for different map symbols. This concern has much to do with each individual's perception and is not trivial by any mean. However, today's cartographic visualization is more concerned with the accuracy of $2 \mathrm{D}$ or $3 \mathrm{D}$ geospatial representations and seeks to accomplish more than simply a straight forward graphic depiction of different features.

Recognition of the power of visualization and graphic modeling, in conjunction with advances in GIS, has led to many advantages in maritime modeling. Examples of these are graphical techniques utilized in applications for marine planning, spatial planning and navigation. Spatial information combined with vessel modeling, is necessary for a full understanding, especially when it comes to training and management context $[6,7]$.

\subsection{The Scene}

The term scene refers to the use of an image as a computer simulation of the currently viewed environment. It can be simply viewed either as a model or a map. The advantages of using the scene are clearly visible when manipulating different models or elements, especially when it comes to making improvements to plans or enhancing the process of crew training.

From 2D photographs to 3D graphical representations, digital vessel representations have developed from abstract and static representations into realistic visualizations, capable of being exported to different formats and explored through dynamic spatial movement [8].

Maritime vessel visualization has addressed many ways to combine maps and plans together into scenes. The 3D graphical tools and technologies enable to create an interactive scene that can be viewed and manipulated in a virtual reality environment. There is much to be gained by bringing research on modeling and visualization in maritime planning closer together and integrating cartographic visualization, land and seascapes. Such an integration could encourage visualizations, so that both coastal and marine environments are viewed together.

\section{Modeling Maritime Vessels}

As a case point, an appropriate GIS platform for interactive and collaborative vessel can provide users with an adequate set of $3 \mathrm{D}$ visualization capabilities. Bringing GIS modeling to bear on visual representations of maritime vessels could be used to raise awareness and improve crew training and education.

Multidimensional maps and depictions are often viewed in 2D. In some cases, virtual environments require expensive stereoscopic glasses combined with special software to view maps and scenes in true $3 \mathrm{D}$, using stereo displays and head tracking mechanisms [9, 10]. However, some capabilities can be used without 
any additional special glasses, making them simply obsolete.

There are many tools that engineers and graphic designers can use to facilitate marine vessel models in cooperation with marine and coastal planning and management, e.g. monitoring cargo containers [11]. These activities may revolve around data generation and presentation, and will benefit significantly from the use of advanced modeling tools.

\subsection{Modeling Tools}

Visual modeling tools and methods should make information available and interpretable to a variety of audiences. They should be suitable for different planning, training and management tasks at hand. Several software tools are also available for visualizing sources of activity in the brain [12]. These techniques include GIS platforms and are cutting edge efforts to link between planning and coastal zone management activities.

Those platforms can allow anyone with a standard PC (Personal Computer) to actively participate in marine and coastal planning efforts. As time goes by, applications that utilize GIS are becoming more available, intuitive and user-friendly.
These services are quite basic, they simply allow different layers of information to be loaded and displayed. By turning a layer on or off, such a system can inform users about what exists and where. They can include floors, walls, sealings, lifts and stairs, as well as entries and exits. By processing information taken from GIS layers, a recommended option or group of options is displayed. An example used for collaborative planning design is shown in Figs. 1-4. It allows users to, e.g. initiate a project by defining the orientation and point of origin, define layers for each vessel element and show the mesh grid of all points and intersections, taking into account the transparency of a model.

When choosing the type of application to use, whether to utilize existing software or develop an application, depends on the resources available and the ultimate goals of a planning process. In this case, user participation is very important. Therefore it would be wise to weight all available options, starting with defining possible users and their particular needs. This can be done by carefully analyzing all existing options and simply designing a set of so-called user cases. User cases are frequently employed for GUI (Graphical User Interface) design of software applications.

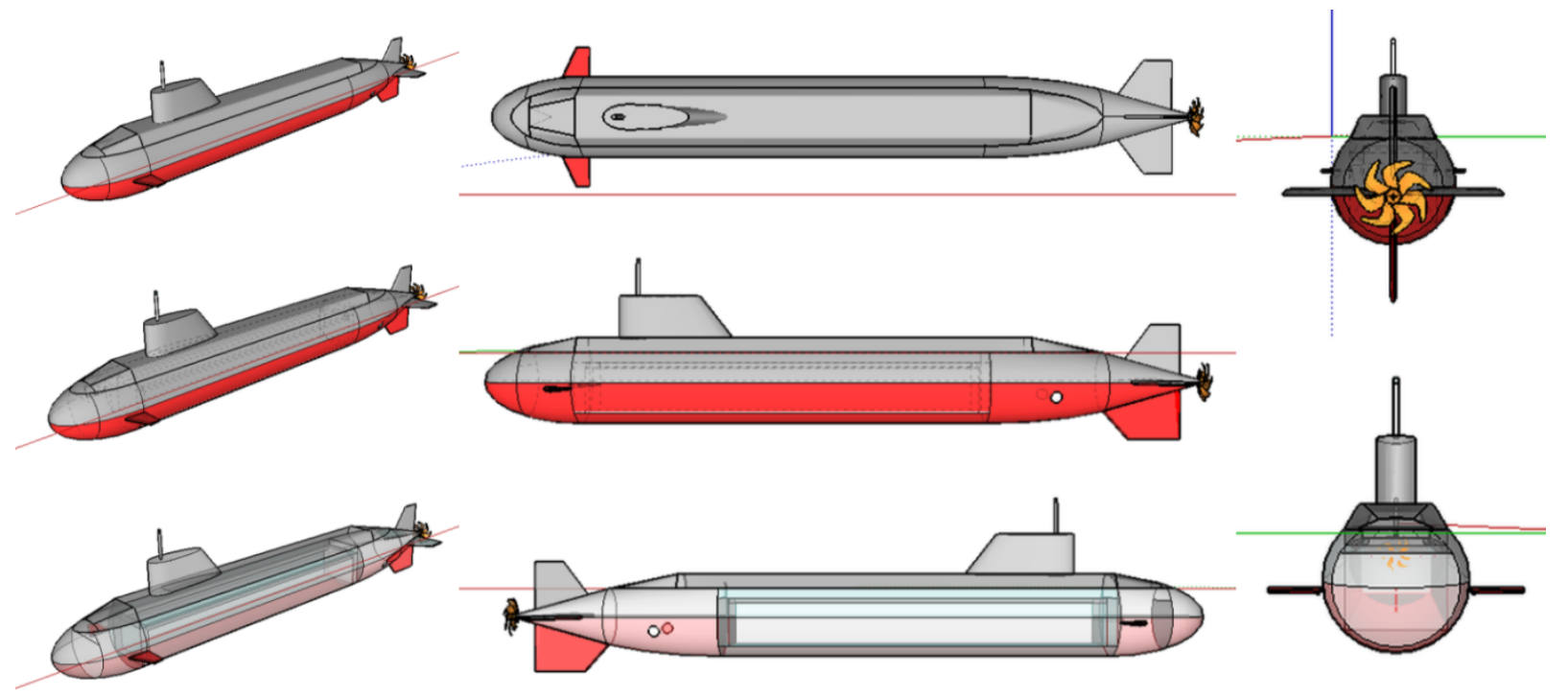

Fig. 1 Submarine 3D model. 


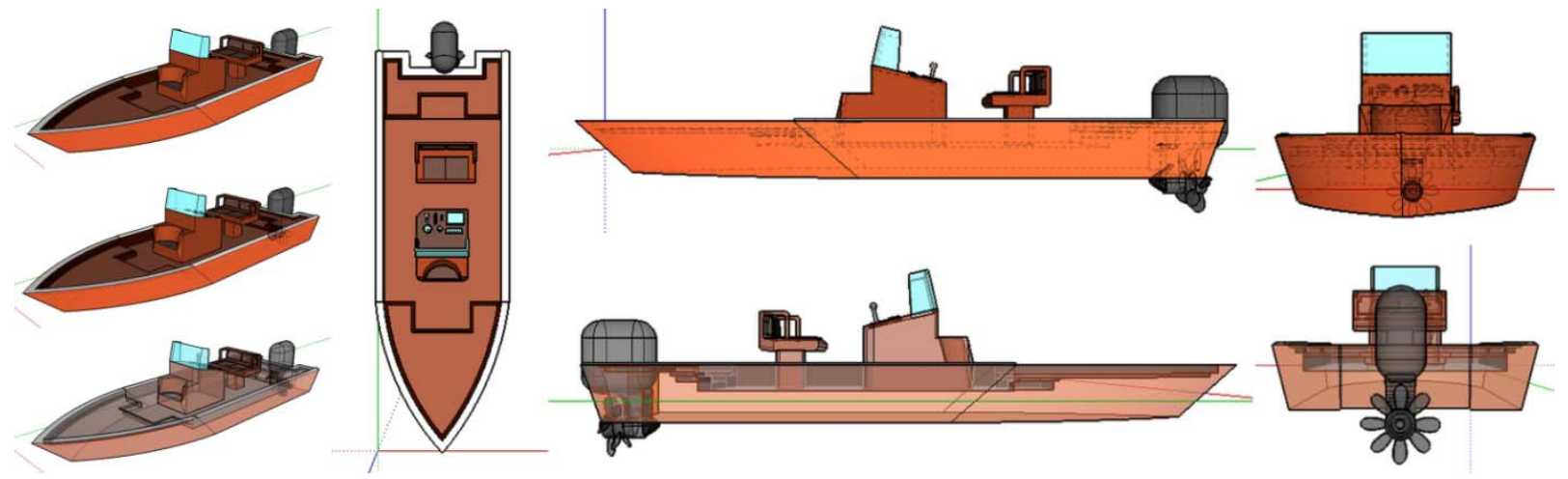

Fig. 2 Boat 3D model.
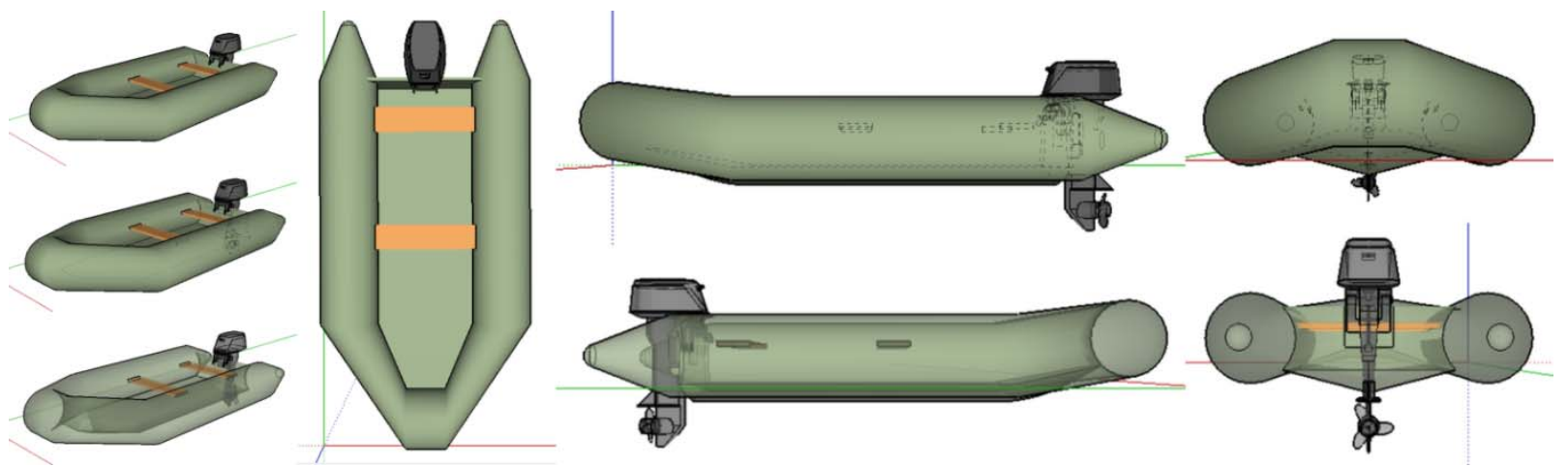

Fig. 3 Pontoon 3D model.
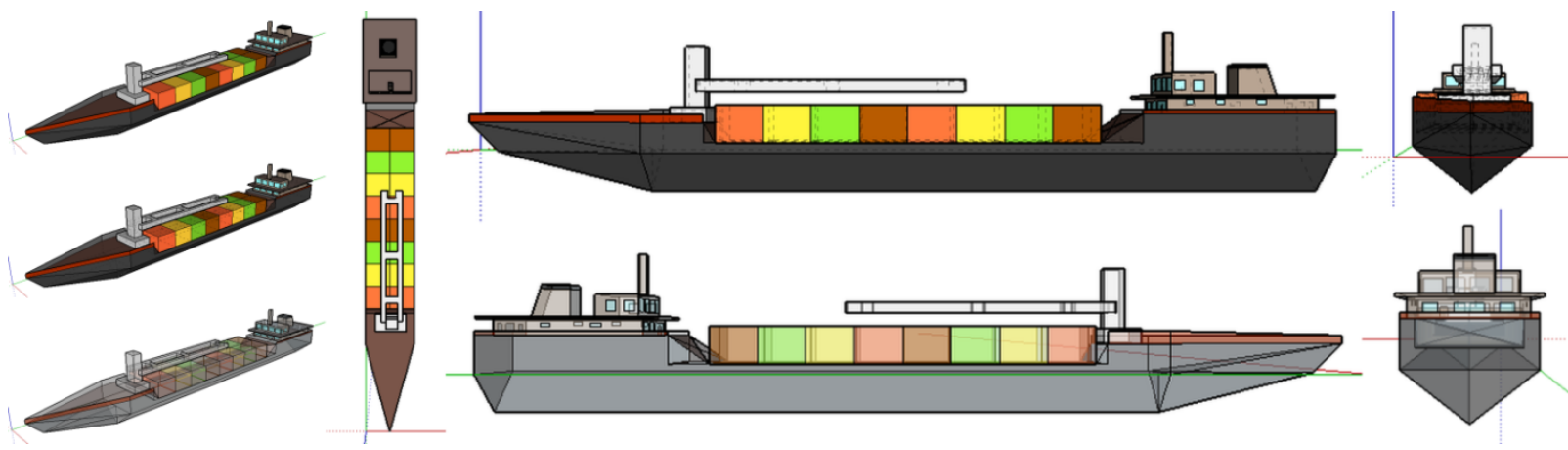

Fig. 4 Container ship 3D model.

\section{The Virtual Environment}

Visualization techniques have been first utilized by using physical models, later through drawing and painting. In those days, only perspective drawings were used. Currently, virtual environments are becoming cutting-edge tools for simulating maritime vessels, involving theater-like laboratories. The advantage of such techniques is its availability and flexibility. The real physical presents of some objects, e.g. a submarine, pontoon, container ship or other vessels is either too expensive, impractical, out of range or currently out of reach for many reasons.

When thinking about training, teaching or education, audiences will often forget information observed in the form of plain text or graphs. Encountering a more real experience through visualization techniques may be less forgettable. Different multimedia, including sound and other visual effects, can also affect the user experience. This combination can be connected to spatial and temporal displays of maps and other additional data representations. 
Conventional real ship practical training on the sea is expensive, high risk and difficult. There is little opportunity for practicing these skills in real-world situations. On the other hand, simulator training is low cost, flexible and repeatable, with methods that provide the opportunity to practice these skills under realistic, yet much safer conditions [13].

\subsection{The Craft of Visualization}

Scientific visualization is a way of using computer graphics to create visual images, which aid in the understanding of complex and often massive numerical representations of scientific concepts or results. Visualization is essential in interpreting data for many scientific problems. It transforms numerical data into a visual representation which is much easier to understand for humans.

The process of data visualization can be described as a sequence of four fundamental processing steps:

(1) Simulation-results of numerical simulations (measurements) are the input of the visualization pipeline.
(2) Data selection and filtering - relevant regions of the raw data are selected, filtered and enhanced. Techniques such as down-sizing, noise filtering and segmentation may be used.

(3) Mapping - the processed data have to be mapped (transformed) into graphical primitives such as points, lines, planes or meshes, and their properties including color, texture and opacity.

(4) Rendering - finally, the graphical primitives are rendered as images, which are then displayed on the screen.

The visualization and representation of objects is not a new concept. It is based on analytical definition techniques of different 3D surfaces and layers, which define the structure of a particular vessel. The use of $3 \mathrm{D}$ visualization for modeling complex objects, including buildings or vessels, has become an important area of research and development of new methodologies [14].

The most widespread techniques for addressing the display of 3D models are based on meshes, so that the computation and storage of data is performed through

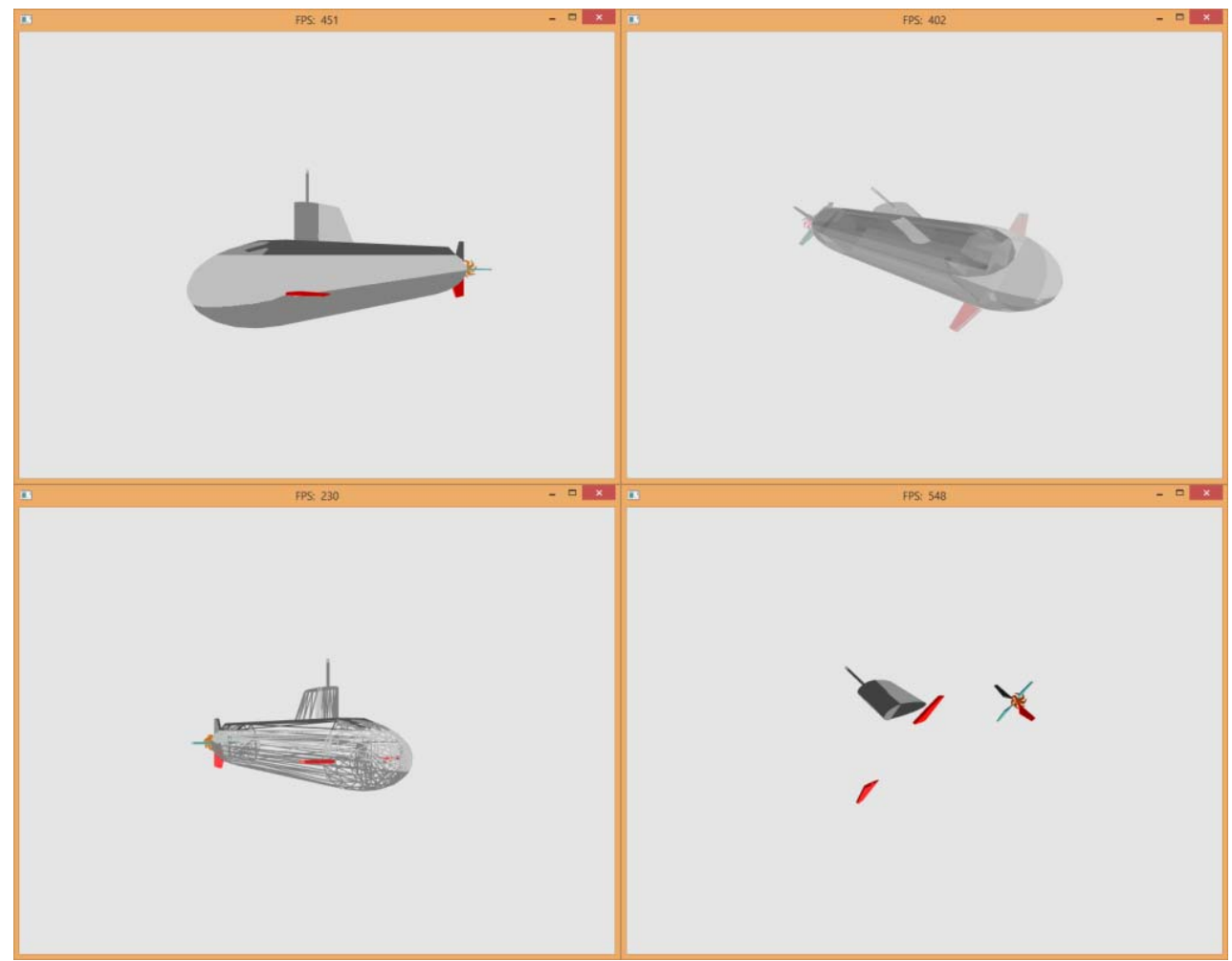

Fig. 5 Visualization of a maritime vessel model. 


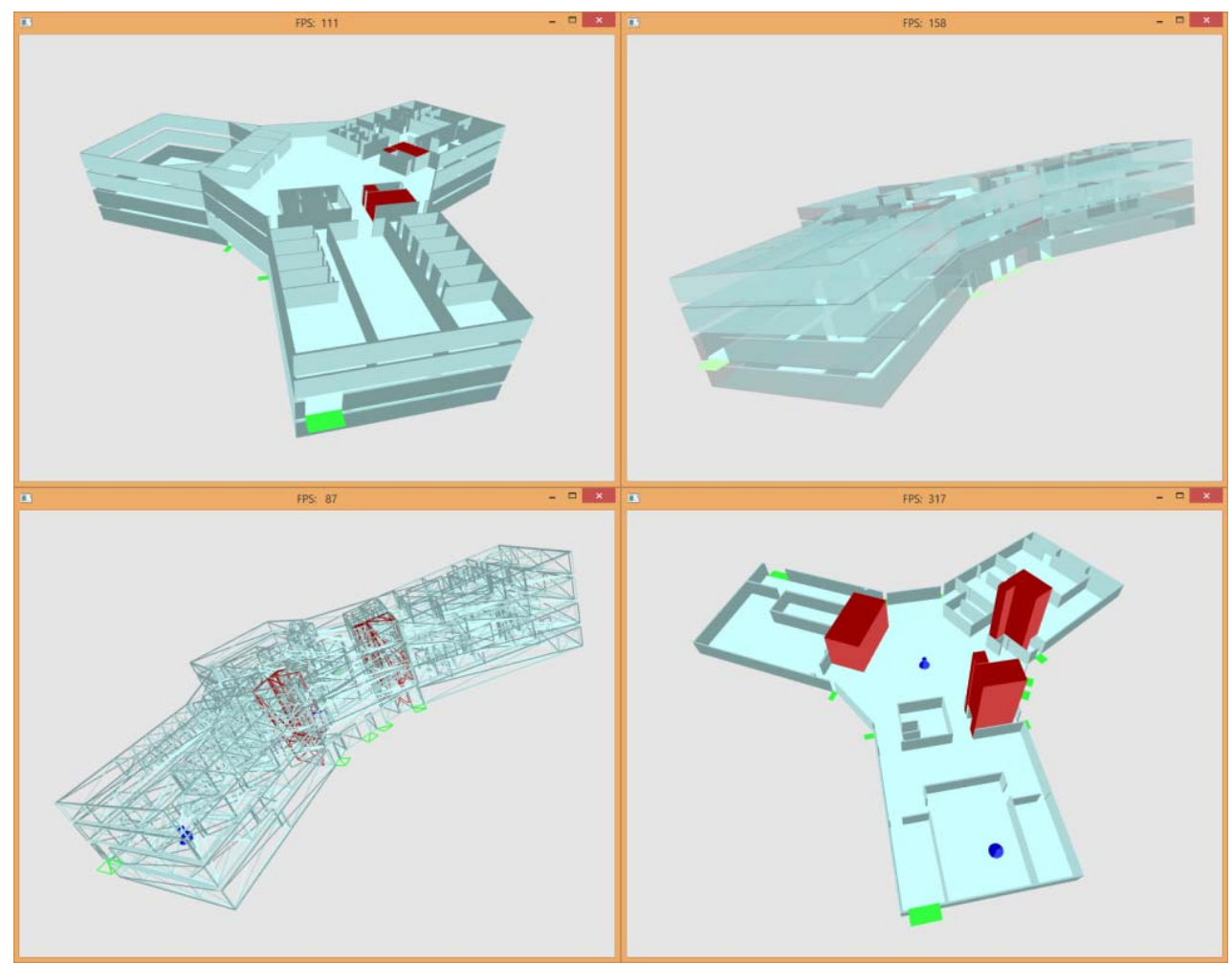

Fig. 6 Visualization of a coastal building model.

all volumes of the working domain. Processing with graphic cards makes it necessary to transform a model into a regular polygon structure. This effective methodology can be also used as a convenient way of building 3D maritime models from available data.

During the animation run it is possible to move, rotate and zoom the 3D model. This feature may be especially useful when recording movies. Other controls and features related to visualization, including adjusting the transparency level and texture visibility, may also be changed in real-time, as shown in Figs. 5 and 6.Of course, virtual reality can be defined in many ways, which all come down to the definition of the significant relationship between humans and computers. It can be described as a simulation of a graphical world with a substantial realistic view. The artificial world is not a static drawing. It can respond to the user, providing a sense of touch and feel. Studies go in that direction to design additional sensors for hearing, taste and even smell [15].

\section{Conclusion}

Maritime maps and plans do not only represent reality, but also create it. This is a good example of how conventional training and education could be pushed forward by the use of advanced tools of visual communication. Such collaboration requires using modeling, computer graphics and computer programming skills to understand. The generic process for developing smart models includes data collection and a combination of hardware and software mechanisms and resources, and finally a user interaction with the system.

In addition to dilemmas about what techniques to use for designing and visualizing model data, engineers are often faced with the question of how to meet the user's expectations, regarding different audiences. In order to be effective, smart modeling requires more than just transferring information to the user. Interaction with the virtual environment is an essential characteristic. Much work has been carried out, but the question for a truly intuitive and natural interaction technique is still 
going on. Users must be able to navigate through the 3D space and manipulate with objects in a user-friendly way.

Both the visual scene and modeling of maritime vessels are fields of study that need more research. More work simply needs to be done on infusing these two types of media. Research on integrating these media could bring better results and encourage training and education of students. After all, combining science and education is one of the main challenges in keeping up with today's labor market requirements. Some would also say that engineers have a moral obligation to promote the use of best practices, technologies and capabilities available.

\section{Acknowledgements}

This paper has been founded by the Polish National Centre for Research and Development (NCBiR) project No. DOBR-BIO4/058/13045/2013.

\section{References}

[1] Portman,M. E. 2014. "Visualization for Planning and Management of Oceans and Coasts." Ocean \& Coastal Management (98): 176-85.

[2] Hansen,A., and Machin, D. 2013."Researching Visual Environmental Communication." Environmental Communication 2(7): 151-68.

[3] Jakubowicz, K. 2009. "A New Notion of Media?" 1st Council of Europe Conference of Ministers Reponsible for Media and New Communication Services Reykjavik, Iceland.

[4] Jarvenpaa, S. L., and Staples, D. S. 2000. "The Use of Collaborative Electronic Media for Information Sharing: an Exploratorystudy of Determinants." Journal of Strategic Information Systems (9): 129-54.

[5] Berson, I. R., Berson, M. J., Desai, S., Falls, D., and
Fenaughty, J. 2008. “An Analysis of Electronic Media to Prepare Children for Safe and Ethical Practices in Digital Environments." Contemporary Issues in Technology and Teacher Education 8(3): 222-43.

[6] Chias, P., and Abad, T. 2013. "Wind Farms: GIS-based Visual Impact Assessment and Visualization Tools." Cartography and Geographic Information Science 3(40): 229.

[7] Orland, B. 1992. "Data Visualization Techniques in Environmental Management: AWorkshop." Landscape and Urban Planning 4(21): 237-9.

[8] Phadke, R. 2010."Steel Forests or Smoke Stacks: The Politics of Visualisation in the Cape Wind Controversy." Environmental Politics 1(19): 1-20.

[9] Popovski, F., Nedelkovski, I., Mijakovska, S., and Ackovski, A. 2015. "Virtual Environment of Real Sport Hall and Analyzing Rendering Quality." TEMJournal 1(4): 112-5.

[10] Oculus Rift website [Online], https://www.oculus.com/, [access: 30.08.2015].

[11] Katulski, R. J., Sadowski, J., Stefański, J., Ambroziak, S. J., and Miszewska, B. 2009. "Self-Organizing Wireless Monitoring System for Cargo Containers." Polish Maritime Research 3(61): 45-50.

[12] Blinowski, G., Kamiński, M., and Wawer, D. 2014. "Trans3D: A Free Tool for Dynamical Visualization of EEG Activity Transmission in the Brain." Computers in Biology and Medicine (51): 214-22.

[13] Liu, X. W., Viao, F. B., and Jin, Y. C. 2009. "A Prototype of Marine Search and Rescue Simulator." International Conference on Information Technology and Computer Science (1): 343-6.

[14] Lazaro, J. M., Navarro, J. A. S., Gil, A. G., and Romero, V. E. 2014. "3D-geological Structures with Digital Elevation Models Using GPU Programming." Computers \& Geosciences (70): 138-46.

[15] Davis, A. 2013. "Smell-O-Vision: Coming to a Living Room Near You?" The Institute [Online] http://theinstitute.ieee.org/technology-focus/technology-t opic/smellovision-coming-to-a-living-room-near-you [access: 30.08.2015]. 